\title{
Carotid bodies and breathing in humans
}

The first report to describe the carotid bodies ("ganglion minutem") was a dissertation published in 1743 by Taube, although initially there was some uncertainty as to whether it was Taube or his professor (Professor Haller who used the term "ganglion exiguum") who was the actual discoverer.* While other investigators continued to report that they had "discovered" these organs (now termed the "ganglion intercaroticum") even as late as the middle of the 19th century, it remained for de Castro's detailed anatomical characterisation to provide the great advance in the understanding of the structures. While he did not actually use the term "chemoreceptors" as such, it is hard to believe that he had anything else in mind when he stated ${ }^{2}$ that they function to "taste the blood". The Nobel Prize winning work of Heymans and his associates in the late 1920s and early 1930s demonstrated their chemoreceptor function unequivocally. ${ }^{3}$ Since then, of course, there have been enormous advances in characterising the carotid bodies with respect to their structure and innervation; functional stimuli, and mechanisms of stimulation; stimulus response characteristics; efferent modulation and integration of the chemosensory information (transmitted centrally via the nerve of Hering and the glossopharyngeal nerve) with other sources of afferent information, predominantly in respiratory and circulatory control.

In considering the role of the carotid bodies in human ventilatory control and blood gas and acid-base regulation, we shall, where possible, consider evidence available from studies on humans, and only consider results from studies on experimental animals when they provide an insight into the control mechanisms that are unattainable in humans.

The major role of the carotid bodies in humans is to defend blood gas and acid-base status in response to real stressors such as lung disease, high altitude, muscular exercise, and sleep, and also the often contrived stimuli for example, hyperoxic hypercapnia - presented by scientists who investigate their control behaviour.

In humans glomic tissues other than the peripheral chemoreceptors, such as the glomus trigeminale, the glomus jugulare, the glomus pulmonare, and the intrapulmonary neuroepithelial bodies, subserve no discernible role in ventilatory responsiveness to hypoxia. Consequently, when the ninth and tenth cranial nerves were blocked by the local anaesthetic lignocaine, the normal hyperventilation which resulted from three minutes of $8 \%$ oxygen breathing was entirely abolished. ${ }^{4}$ Interestingly, however, there was also no evidence of ventilatory depression during this time. Although the authors interpreted this as suggesting that their anaesthetic blockade may not have been complete, there was, in fact, no evidence of an early hyperpnoea followed by a subsequent reduction in ventilation. However, the aortic bodies in humans (unlike the cat and dog, for example) appear to subserve little, if any, functional role in hypoxic ventilatory responsiveness - that is, in response to reductions in arterial oxygen partial pressure $\left(\mathrm{PaO}_{2}\right)$. It also seems unlikely that they are important in transducing reductions in oxygen content, such as those that result from anaemia or carboxyhaemoglobinaemia in humans - responses that have been

* The author is grateful to Dr Benjamin Winter for drawing these historical references to his attention. clearly demonstrated in the cat. ${ }^{5}$ The carotid bodies are therefore the organs which subserve hypoxic ventilatory responsiveness in humans; consequently, they play a vital part in constraining the fall of arterial $\mathrm{Po}_{2}$ in response to the challenge of hypoxaemia.

The most convincing evidence supporting this contention comes from studies performed in subjects who have had both carotid bodies surgically resected (see below). These subjects have no hyperpnoea in response to experimentally induced hypoxaemia, ${ }^{67}$ nor do they show a decline in ventilation following the abrupt and surreptitious administration of $100 \%$ oxygen against an hypoxic background. ${ }^{7}$ However, in tests in which the alveolar - and hence arterial - partial pressure of carbon dioxide was elevated, Honda $e t a l^{8}$ and Swanson et $a l^{9}$ were able to demonstrate a small component of hypoxic ventilatory responsiveness in such subjects. As increased arterial $\mathrm{PCO}_{2}$ is known to increase peripheral chemosensitivity to hypoxia, it is likely therefore that the aortic bodies in humans subserve a component of hypoxic ventilatory drive that is normally too small to be discerned but which is demonstrable when their sensitivity is amplified. It is not impossible, however, on the basis of these experiments, to rule out regeneration of the nerve endings in Hering's nerve, which has been shown in the cat and which might, under these conditions, be capable of hypoxic sensory transduction.

It is also known that ventilation in normal subjects responds typically in a biphasic manner to an acute hypoxic stimulus - that is, following a square wave induction of alveolar hypoxia in humans there is an early rapid hyperpnoea which is followed after a few minutes by a subsequent decline in ventilation to a new stable level. This profile is seen even when alveolar $\mathrm{PCO}_{2}$ is clamped at a constant level throughout the test by manipulating the inspired carbon dioxide fraction. The rapid increase is considered to be attributable to carotid chemosensitivity; the subsequent decline is a reflection of a reduction in central ventilatory drive (often termed "depression"). The secondary decline has been attributed both to increased cerebral blood flow washing carbon dioxide out of the cerebral interstitial fluid, and also by the influence of central neurotransmitters such as GABA and possibly adenosine which decrease ventilation. Interestingly, Khamnei and Robbins ${ }^{10}$ have proposed that this mechanism may be more complex, requiring input from the carotid bodies. Support for this notion may be found in the finding by Honda ${ }^{11}$ and in our own laboratory (S A Ward and BJ Whipp, unpublished data) that there was no evidence of hypoxic ventilatory depression in response to the square wave induction of an hypoxic stimulus (alveolar $\mathrm{Po}_{2} \sim 50-55$ Torr or $7 \cdot 0-7 \cdot 5 \mathrm{kPa}$ ) of 10 minutes or more in subjects without carotid bodies. Whether such depression would occur with more severe hypoxia is, of course, uncertain.

The role of the carotid bodies in more prolonged hypoxia is more complex. During the acclimation phase to high altitude the sensitivity of the carotid bodies increases progressively over a period of weeks. ${ }^{12}$ This, in concert with the reduction in the alkaline constraint of ventilation as renal and cerebrospinal fluid (CSF) bicarbonate excretion increases, explains the further increase in ventilation and greater hypocapnia that is evident over this time course in 
lowlanders sojourning at high altitude. The acclimation, however, can occur even when the hypoxia is localised to the carotid bodies. ${ }^{13}$ Efferent activity in the nerve of Hering is also important in modulating carotid body hypoxic responsiveness. Conditions such as CSF alkalaemia, which increases impulse traffic in the efferent fibres of Hering's nerve, suppress afferent nerve traffic from the carotid bodies, and hence their functioning should be considered to be part of an integrated peripheral and central response to respiratory stimulation. Subjects born and resident at high altitudes often have considerably reduced hypoxic ventilatory responsiveness, despite hypertrophy of the carotid bodies. There are also suggestions that chronic hypoxia in subjects with chronic obstructive pulmonary disease (COPD) may lead to a reduced ventilatory response to hypoxia. However, such studies are complicated by the increased airways resistance in these subjects which constrains the ventilatory response.

We are aware of no systematic studies which have looked at the magnitude of the decrease in ventilation when subjects with COPD are caused to breathe pure oxygen abruptly to suppress carotid chemosensitivity. However, there is evidence which suggests that such chronically hypoxic subjects do not entirely lose their peripheral chemosensitivity. For example, subjects with severe COPD who have undergone bilateral carotid body resection show a further hypoxaemia that is consistent with the removal of an ongoing hypoxic drive as a result of the surgery. ${ }^{1415}$

The carotid bodies also have an important role in regulating blood gas and acid-base status during muscular exercise; this, of course, includes the sudden increases in metabolic and ventilatory demands encountered during the activities of daily living. These organs do not contribute discernibly to the initial hyperpnoea which occurs during the transit delay from the exercising limbs to the lungs (phase I): the magnitude of phase I has been shown to be unaltered with either hypoxic or hypercapnic inspirates and is also normal in subjects without carotid bodies. ${ }^{16}$ In normal euoxic subjects the carotid bodies have been shown to contribute, on average, approximately $20 \%$ of the ventilatory drive in the steady state of exercise (phase III). This proportion does not seem to vary appreciably with work rate, even when associated with a metabolic (chiefly lactic) acidosis (that is, above the lactate threshold). The absolute contribution of the carotid bodies to the exercise hyperpnoea consequently increases as a function of work rate. The increase in hypoxic ventilatory responsiveness which has been seen during exercise is mediated by a mechanism that seems to depend more on the increase in metabolic rate than on the exercise itself. The magnitude of the exercise hyperpnoea, ${ }^{17} 18$ however, does not correlate well with resting indices of peripheral chemosensitivity. ${ }^{19}$

Considerable recent work has shown that increased arterial $\left[\mathrm{K}^{+}\right]$plays an important part in the carotid body component of the hyperpnoea. ${ }^{20}$ However, other known carotid body stimuli also increase in arterial blood during exercise, including adenosine, osmolarity, and the rate of change of the arterial $\mathrm{pH}$ oscillation; $\mathrm{pH}$, of course, also decreases when there is an associated metabolic acidosis. The relative importance of these stimuli in isolation and, the characteristics of their interaction remains to be determined. The combination of exercise and hypoxaemia, whether as a result of reduced inspired $\mathrm{Po}_{2}$ or of lung disease, can result in the carotid bodies contributing more than $50 \%$ of the ventilatory drive, ${ }^{21}$ even at a relatively modest $\mathrm{PaO}_{2}$ of 55 Torr $(\sim 7.5 \mathrm{kPa})$. We are aware of no such data with more extreme hypoxaemia.

The carotid bodies appear to subserve an even greater role in the non-steady state phase of the hyperpnoea (phase II). This, of course, is the more common feature of normal activity. The time course of the exponential increase in ventilation in this phase correlates closely with that of pulmonary carbon dioxide output (not, it should be noted, with tissue carbon dioxide production rate). Furthermore, pulmonary carbon dioxide output changes appreciably more slowly than oxygen uptake in this phase as a result of the high tissue capacitance for carbon dioxide. Consequently, ventilation lags the oxygen uptake changes significantly during the transient. The time constant of the responses are approximately $60-70$ seconds and 30-40 seconds, respectively. As a result there is a transient hypoxaemia during phase II even in normal subjects.

It has been shown, however, that the phase II ventilatory time constant is highly dependent upon the gain or sensitivity of the carotid bodies. ${ }^{21}$ Reduced sensitivity, such as that which results from induced hyperoxia, metabolic alkalaemia, intravenous infusion of dopamine, or carotid body resection, causes a further slowing of ventilation. Increased sensitivity, as a result of hypoxaemia or metabolic acidosis, speeds the time constant of the ventilatory response. The oxygen uptake time constant is not appreciably altered, however, by these interventions. The speeding of the ventilatory time constant as a result of the increased carotid chemosensitivity ameliorates the further hypoxaemia of exercise in a subject who is already hypoxaemic. This reflects an important and under appreciated control function of the carotid bodies. However, these studies have been carried out almost entirely in normal subjects at sea level. The control characteristics with, for example, the chronic hypoxaemia of lung disease, in high altitude natives and in highly trained athletes remain to be determined.

The carotid bodies play a significant part in the compensatory hyperventilation which contains the fall of arterial $\mathrm{pH}(\mathrm{pHa})$ at work rates which induce a metabolic acidosis. The fact that subjects with myophosphorylase deficiency (McArdle's syndrome) can hyperventilate and develop a respiratory alkalaemia at high work rates does not, of course, undermine this contention. Normal subjects breathing high inspired oxygen fractions, ${ }^{21}$ and subjects (with relatively normal pulmonary mechanics and blood gas tensions) who have had both carotid bodies surgically resected, ${ }^{16}$ demonstrated significantly slowed compensatory hyperpnoea to a given change of blood levels of lactate and standard bicarbonate. The fall of $\mathrm{pHa}$ was consequently significantly greater. Hypoxia resulted in a greater and more rapid compensatory hyperpnoea, causing the fall of $\mathrm{pHa}$ to be much smaller. ${ }^{21}$ Although there appears to be a slow compensatory mechanism which is independent of the carotid bodies (possibly a slow transport of $\mathrm{H}^{+}$into the brain extracellular fluid), these chemoreceptors appear to subserve an important role in response to acute metabolic acid-base challenges.

Although unilateral carotid body resection as a "treatment" for bronchial asthma was reported by Nakayama and $\mathrm{Seo}^{22}$ in 1942 and by Legatte ${ }^{23}$ in 1950, it was not until 1961 that significant interest in the procedure was aroused. In that year Nakayama ${ }^{24}$ and Overholt ${ }^{25}$ both published the results of unilateral resection in the American literature. The results of this procedure subsequently proved to be highly variable and this led to a wide range of opinion being reported, both supportive and condemnatory. The procedure was consequently largely discarded. Wood and colleagues ${ }^{26}$ reported that bilateral carotid body resection reduced asthmatic symptoms. An unfortunate consequence of this removal of carotid body function was the loss of carotid baroreceptor function with its untoward consequences for baroregulation. However, this also often occurred with the inadvertent loss of function as a result of carotid endarterectomy. 
Winter, ${ }^{27}$ however, discovered that the baroreceptor and chemoreceptor components of the carotid bifurcation nerve supply largely separated into two functionally distinct tracts before joining into the common trunk as the nerve of Hering in humans. He further showed that it was possible to remove the carotid bodies in humans without significantly disrupting the baroreceptor afferents. This was subsequently supported by the demonstration that arterial blood pressure was normal both at rest and at several levels of muscular exercise, and that the cardiovascular responses to carotid sinus massage and a Valsalva manoeuvre were normal. ${ }^{28}$ Winter also proposed, however, that the procedure might be beneficial to patients with advanced chronic obstructive airways disease, in addition to those with bronchial asthma, as it reduced dyspnoea. He also reported that bilateral carotid body resection reduced both functional residual capacity and residual volume and increased expiratory airflow (as evidenced by an increased $\mathrm{FEV}_{1}$ ) in patients with COPD.

The ostensive physiological basis of these improvements and, in fact, a reported justification for the procedure was the finding by Nadel and Widdicombe, in the 1960s, that the carotid bodies, in addition to their role in ventilatory control, also formed a component of a reflex bronchomotor loop ${ }^{29}$ - that is, stimulation of the carotid bodies in the dog led to airway constriction. This could be obviated by section of the glossopharyngeal or the vagus nerves - that is, the afferent and efferent components of this reflex loop. It was quite clear, however, from the earliest studies of physiological function following carotid body resection that there was a loss of hypoxic ventilatory responsiveness. The absence of this control component was proposed to be deleterious to patients who were already hypoxaemic, and would also be expected to exacerbate any concomitant cor pulmonale.

Support for the controversial procedure and, in fact, its continued performance rested almost exclusively with Winter. Editorials by prominent physicians and physiologists in major medical journals ${ }^{3031}$ concluded, on the one hand, that the loss of carotid body function was of relatively minor consequence, and to the other extreme, that their removal could result in significant physiological impairments that were potentially hazardous to the patients.

Studies of the physiological consequences of bilateral carotid body resection (that is, pre- and post-surgery studies in the same subjects) reveal: an on-average small hypoxaemia and hypercapnia - but with large individual variability - as a result of the procedure ${ }^{1415}$; loss of acute hypoxic ventilatory responsiveness; reduction of the euoxic carbon dioxide ventilatory responsiveness of the order of $20-30 \%$; loss of the hypercapnic potentiation of hypoxic ventilatory drive; and reduction of dyspnoeic sensation. While there appears to be no debate that carotid chemostimulation contributes to the perception of shortness of breath, ${ }^{15273031}$ differences remain as to whether carotid body stimulation contributes solely as a function of the consequent hyperpnoea or proportionally more than other sources of ventilatory stimulation. Ward and Whipp, ${ }^{32}$ using an isopnoeic technique, support the role of the carotid bodies as an especially potent source of dyspnoea while Lane $e t a l{ }^{33}$ using progressively increasing ventilatory profiles, support the concept that the resulting dyspnoea is merely a function of the consequent hyperpnoea rather than being dependent on the afferent source of stimulation. The issue awaits resolution.

As a consequence of the absence of the carotid body mediated component of dyspnoea, however, breath-hold time (BHT) is appreciably longer in subjects with bilateral carotid body resection than in either normal subjects or asthmatic controls. ${ }^{34}$ This was especially true with hypoxic inspirates (inspired oxygen fraction $=0 \cdot 12$ ) for which subjects wth bilateral carotid body resection had no significant reduction in BHT compared with the air breathing value (BHT $\simeq 125$ seconds). In normal subjects, however, BHT fell from an air breathing average of 75 seconds to 60 seconds with the hypoxic inspirate. There was, however, no significant difference between the normal subjects and those with bilateral carotid body resection when the inspired oxygen fraction was 1.0 - that is, consistent with the normal group having no functional carotid chemosensitivity with this degree of hyperoxia.

The steady state ventilatory response to muscular exercise was shown to be appropriate to regulate arterial blood gas and acid-base status in asthmatic subjects following bilateral carotid body resection ${ }^{7}$ who had little or no abnormalities of blood gas and pulmonary mechanical function. Subjects with airflow limitation, however, showed carbon dioxide retention during exercise following bilateral carotid body resection. ${ }^{35}$ There is clear evidence, however, that there is a slowing of the time course of ventilation towards the steady state following bilateral carotid body resection. ${ }^{1621}$ This resulted in a transient respiratory acidaemia and, unlike normal subjects in whom hypoxia potentiates the ventilatory response to exercise, there was no potentiation in the carotid body resected group. ${ }^{7}$ In addition, there was considerable slowing of the ventilatory response to the metabolic acidosis of exercise. ${ }^{16}$ Consequently, rather than the partially compensated metabolic acidaemia of exercise which results in normal subjects, those without carotid bodies showed a combined metabolic and respiratory acidosis at work rates above the lactate threshold.

Responses to incremental exercise before and after bilateral carotid body resection were also studied in a group of patients with COPD. ${ }^{14}$ The results showed that, following bilateral carotid body resection, the subjects were able to exercise to a higher work rate and consequently a higher oxygen uptake. Interestingly, however, the same maximum level of ventilation during the exercise was unchanged. This is consistent with respiratory limitation of the exercise both before and after bilateral carotid body resection but with a component of the drive to breathe - that is, the hypoxic and metabolic acidaemic components - being removed, so that a higher work rate could be performed before the maximum and limiting level of ventilation is attained. The consequence, therefore, was a further hypoxaemia and hypercapnia in these patients.

Both Vermeire and colleagues ${ }^{15}$ and Whipp and Ward ${ }^{14}$, who studied the effects of bilateral carotid body resection on pulmonary mechanics in patients with COPD, reported an improvement in lung mechanics following the procedure. Plethysmographically determined airways resistance was reduced and $\mathrm{FEV}_{1}$ increased by approximately $9 \%$. While this is suggestive of the bronchomotor reflex shown in the dog by Nadel and Widdicombe, ${ }^{29}$ it is not compelling since other factors such as arterial and alveolar gas tensions and changes in mucus output (and possibly rheology) might also influence these measurements. Although concerns were raised over the implications for mortality, the available evidence suggests that the mortality rates are not increased as a result of bilateral carotid body resection. ${ }^{1536}$ There was also no evidence of significantly altered sleep structure or frequency of nocturnal haemoglobin desaturation, as shown by nocturnal polysomnographic studies, ${ }^{15}$ in a small group of patients after bilateral carotid body resection.

It is generally conceded that dyspnoea, or at least a component of dyspnoea, is relieved by the procedure, and consequently many patients have reported an improvement 
in life style. Some others, however, have reported dissatisfaction with the procedure. The question remains, therefore, whether the relief of dyspnoea afforded by bilateral carotid body resection might be considered to outweigh any deleterious physiological effects in some cases. If so, what criteria might be applied to select those particular patients - assuming of course that other medical managements have proven ineffective?

\section{Department of Physiology,}

St Geonge's Hospital Medical School,

B J WHIPP

Cranmer Terrace,

London SW17 ORE,

$U K$

1 Taube HWL. De vera nervi intercostalis origine. Inaug Diss. Gottingae: A Vanderhoeck, 1743.

2 De Castro F. Sur la structure et l'innervation de la glande intercarotiddienne (glomus caroticum) de l'homme et des mammiferes, et sur un nouveau système d'innervation autonome du nerf glossopharyngien. Ėtudes anatomiques et espérimentales. Trabajos Lab Invest Biol Univ Madrid $1926 ; 24: 365-432$.

3 Heymans C, Bouckaert J-J, Dautrebande L. Sinus carotidien et réflexes respiratoires. II. Influences respiratoires réflexes de l'alcalose, de l'anydride carbonique, de l'ion hydrogene et de l'anoxemie. Sinus carotiden et echanges respiratoires dans les poumons et ou dela des poumons. Arch Int Pharmacodyn 1930;39:400-50.

4 Guz A, Noble MIM, Widdicombe JG, Trenchard D, Mushin WW. Peripheral chemoreceptor block in man. Respir Physiol 1966;1:38-40.

5 Lahiri S. Oxygen biology of peripheral chemoreceptors. In: Lahiri S, Cherniack NS, Fitzgerald RS, eds. Response and adaptation to hypoxia. Cherniack NS, Fitzgerald RS, eds. Response and

6 Holton P, Wood JB. The effects of bilateral removal of the carotid bodies and denervation of the carotid sinuses in two human subjects. $\mathcal{F}$ Physiol 1965;181:365-78.

7 Lugliani R, Whipp BJ, Seard C, Wasserman K. Effect of bilateral carotidbody resection on ventilatory control at rest and during exercise in man. N Engl F Med 1971;285:1105-11.

8 Honda Y, Watanabe S, Hashizume I, Satomura Y, Hata N, Sakakibara Y, et al. Hypoxic chemosensitivity in asthmatic patients two decades after carotid body resection. F Appl Physiol 1979;46:632-8.

9 Swanson GD, Whipp BJ, Kaufman RD, Aqloh KA, Winter B, Bellville JW. Effect of hypercapnia on hypoxic ventilatory drive in normal and carotid Effect of hypercapnia on hypoxic ventilatory drive in
body-resected man. $¥$ Appl Physiol 1978;45:971-7.

10 Khamnei S, Robbins PA. Hypoxic depression of ventilation in humans: alternative models for the chemoreflexes. Respir Physiol 1990;81:117-34.

11 Honda $Y$. Respiratory and circulatory activities in carotid body-resected humans. $\mathcal{F}$ Appl Physiol 1992;73:1-8.

12 Sato M, Severinghaus JW, Powell FL, Xu F, Spellman MJ Jr. Augmented hypoxic ventilatory response in man at altitude. $\mathcal{F}$ Appl Physiol 1992;73: 101-7.
13 Bisgard GE, Busch MA, Forster HV. Ventilatory acclimatization to hypoxia is not dependent on cerebral hypocapnic alkalosis. $\mathcal{F}$ Appl Physiol 1986; 60:1011-5

14 Whipp BJ, Ward SA. Physiologic changes following bilateral carotid-body resection in patients with chronic obstructive pulmonary disease. Chest 1992;101:655-61

15 Vermeire P, de Backer W, van Maele R, Bal J, van Kerckhoven W. Carotid body resection in patients with severe chronic airflow limitation. Clin Respir Physiol 1987;23(Suppl 11): 165-6S.

16 Wasserman K, Whipp BJ, Koyal SN, Cleary MG. Effect of carotid body resection on ventilatory and acid-base control during exercise. $₹$ Appl resection on ventilatory

17 Zwillich CW, Sahn SA, Weil JV. Effects of hypermetabolism on ventilation and chemosensitivity. $\mathcal{f}$ Clin Invest 1977;60:900-6.

18 Pandit JJ, Bergstrom E, Frankel HL, Robbins PA. Increased hypoxic ventilatory sensitivity during exercise in man: are neural afferents necessary? f Physiol 1994;477:169-75.

19 Weil JV, Swanson GD. Peripheral chemoreceptors and the control of breathing. In: Whipp BJ, Wasserman $\mathrm{K}$ eds. Pulmonary physiology and pathophysiology of exercise. New York: Marcel Dekker, 1991:371-403.

20 Paterson DJ. Potassium and ventilation in exercise. $\mathcal{F}$ Appl Physiol 1992;72: 811-20.

21 Whipp BJ. Peripheral chemoreceptor control of the exercise hyperpnea in humans. Med Sci Sports Exerc 1994;26:337-47.

22 Nakayama K, Seo S. Surgery of the carotid body. Proc Tokyo Surgical Meeting $1942 ; 414$

23 Leggate JM. Treatment of asthma by excision of the carotid body. Postgrad Med 1950;26:71-7.

24 Nakayama K. Surgical removal of the carotid body for bronchial asthma. Dis Chest 1961;40:595-604.

25 Overholt RH. Glomectomy for bronchial asthma. Dis Chest 1961;40:605-10.

26 Wood JB, Frankland AW, Eastcott HHG. Bilateral removal of the carotid bodies for asthma. Thorax 1965;20:570-3.

27 Winter B. Bilateral carotid body resection for asthma and emphysema: a new surgical approach without hypoventilation or baroreceptor dysfunction. Int Surg 1972;57:458-64.

28 Lugliani R, Whipp BJ, Wasserman $\mathrm{K}$. A role for the carotid body in cardiovascular control in man. Chest 1973;63:744-50.

29 Nadel JA, Widdicombe JG. Effect of changes in blood gas tensions and carotid sinus pressure on tracheal volume and total lung resistance to air flow. $\mathcal{F}$ Physiol 1962;163:13-33.

30 Severinghaus JW. Carotid body resection for COPD? Chest 1989;95:1128-9. Wasserman K. The carotid bodies: pathologic or physiologic? Chest 1978; 73:564-6.

32 Ward SA, Whipp BJ. Effects of peripheral and central chemoreflex activation on the isopnoeic rating of breathing in exercising humans. $f$ Physiol 1989; 411:27-43.

33 Lane R, Adams L, Guz A. The effects of hypoxia and hypercapnia on perceived breathlessness during exercise in humans. F Physiol 1990;428: 579-93.

34 Davidson JT, Whipp BJ, Wasserman K, Koyal SN, Lugliani R. Role of the carotid bodies in the sensation of breathlessness during breath-holding. $N$ Engl 7 Med 1974;290:819-22.

35 Honda Y, Myojo S, Hasegawa S, Severinghaus JW. Decreased exercise hyperpnea in patients with bilateral carotid chemoreceptor resection. $f$ Appl Physiol 1979;46:908-12.

36 Winter B. Carotid body resection in chronic obstructive pulmonary disease. Chest 1991;100:863. 